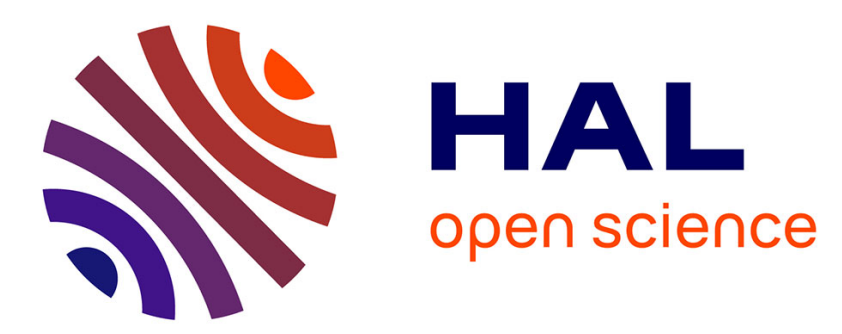

\title{
Détecteur de neutrons rapides à réponse dépendant peu de l'énergie
}

\author{
J.L. Leroy, J.L. Huet, I. Szabo, C. Le Rigoleur, D. Abramson
}

\section{To cite this version:}

J.L. Leroy, J.L. Huet, I. Szabo, C. Le Rigoleur, D. Abramson. Détecteur de neutrons rapides à réponse dépendant peu de l'énergie. Revue de Physique Appliquée, 1969, 4 (2), pp.251-252. 10.1051/rphysap:0196900402025100 . jpa-00243246

\section{HAL Id: jpa-00243246 https://hal.science/jpa-00243246}

Submitted on 1 Jan 1969

HAL is a multi-disciplinary open access archive for the deposit and dissemination of scientific research documents, whether they are published or not. The documents may come from teaching and research institutions in France or abroad, or from public or private research centers.
L'archive ouverte pluridisciplinaire HAL, est destinée au dépôt et à la diffusion de documents scientifiques de niveau recherche, publiés ou non, émanant des établissements d'enseignement et de recherche français ou étrangers, des laboratoires publics ou privés. 


\title{
DÉTECTEUR DE NEUTRONS RAPIDES A RÉPONSE DÉPENDANT PEU DE L'ÉNERGIE
}

\author{
J. L. Leroy, J. L. HUet, I. SZABO, G. LE Rigoleur et D. ABRAMSON, \\ C.E.N., Gadarache.
}

Résumé. - Cet appareil, destiné à des mesures absolues de flux neutroniques, se compose essentiellement d'un bloc de polyéthylène et de deux compteurs à $\mathrm{BF}_{3}$. L'ensemble est entouré d'une protection, et les neutrons sont amenés au cœur du dispositif par un collimateur. On a mesuré l'efficacité du système en valeur absolue vers $0,3 \mathrm{MeV}$ et vers $2,7 \mathrm{MeV}$ et la forme de la courbe de réponse entre $0,1 \mathrm{MeV}$ et $4 \mathrm{MeV}$.

\begin{abstract}
This device, designed with the aim of performing absolute measurements of neutron fluxes, is basically made of polyethylene and two $\mathrm{BF}_{3}$ counters, surrounded by a thick shield. Neutrons to be counted are fed at the center of the device by a collimator. The absolute efficiency around $0.3 \mathrm{MeV}$ and $2.7 \mathrm{MeV}$ has been measured as well as the shape of response curve between 0.1 and $4 \mathrm{MeV}$.
\end{abstract}

I. Description. - Le dispositif est représenté sur la figure 1. Les dimensions du canal d'entrée du collimateur sont choisies en fonction de la distance à la source de façon que les neutrons aillent jusqu'en A. Dans une telle géométrie, contrairement au long compteur habituel, les résonances du carbone n'introduisent pas d'irrégularité appréciable dans la courbe de réponse, et de plus le collimateur et la protection permettent de sélectionner d'une façon plus stricte les neutrons provenant de la source à mesurer.

II. Etude expérimentale des propriétés du système. - II.1. Galibration absolue. - On a eu recours autant que possible à des méthodes directes, faisant intervenir le moins possible de données nucléaires : i) entre $100 \mathrm{keV}$ et $250 \mathrm{keV}$, la calibration a été réalisée par comparaison avec un scintillateur de verre au lithium dont on avait mesuré directement l'efficacité par la méthode de la particule associée avec la réaction $\mathrm{T}(\mathrm{p}, \mathrm{n})^{3} \mathrm{He}$. Le principe de cette expérience a été décrit ailleurs [1]; ii) dans une gamme d'énergie plus élevée, on peut aussi mettre à profit les propriétés de la réaction $d, d$. Le comptage absolu des particules ${ }^{3} \mathrm{He}$ de la réaction $\mathrm{D}(\mathrm{d}, \mathrm{n})^{3} \mathrm{He}$ permettrait de déterminer le flux de neutrons, mais il est très difficile de faire ce comptage avec précision. On peut par contre compter facilement les protons et tritons de la réaction $\mathrm{D}(\mathrm{d}, \mathrm{p}) \mathrm{T}$. Entre $50 \mathrm{keV}$ et $300 \mathrm{keV}$, les deux processus ont des sections efficaces comparables, et le rapport de ces grandeurs, ainsi que les distributions angulaires des deux réactions ont été mesurés avec précision, notamment par Theus et al. [2]. On mesure le flux de protons dans un angle solide défini, et on en déduit le flux de neutrons dans la direction du compteur à étalonner; iii) la méthode du bain de $\mathrm{SO}_{4} \mathrm{Mn}$ [3] a été utilisée également. La cuve était placée dans une protection

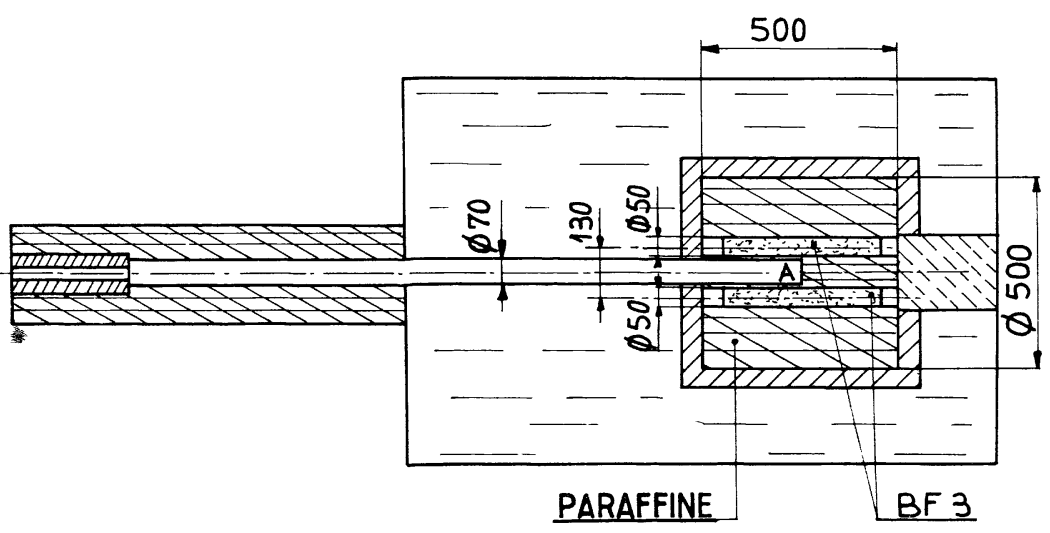

FIG. 1. 
TABLEAU I

$\begin{array}{llllllllll}\text { Énergie }(\mathrm{MeV}) & 0,100 & 0,250 & 0,500 & 2,000 & 2,500 & 3,000 & 3,500 & 4,000 & 4,3 \cap 0 \\ \text { Efficacité } \ldots . . & 0,139 & 0,135 & 0,134 & 0,133 & 0,126 & 0,123 & 0,113 & 0,106 & 0,102\end{array}$

analogue à celle du compteur. Un faisceau quasi parallèle de neutrons était admis au centre de la cuve par un collimateur.

II. 2. VARIATION RELATIVE DE L'EFFIGAGITÉ EN FONGTION DE L'ÉNERGIE DES NEUTRONS. - Elle a été mesurée directement : i) entre 2 et $4,2 \mathrm{MeV}$, on a utilisé la propriété de symétrie de la réaction $\mathrm{D}(\mathrm{d}, \mathrm{n})^{3} \mathrm{He}$. La distribution des neutrons est forcément symétrique par rapport à $90^{\circ}$ dans le système du centre de masse, puisque le projectile et la cible sont identiques. On choisit alors des directions formant des angles supplémentaires dans le système du centre de masse et on calcule les angles qui correspondent dans.le système du laboratoire ainsi que les énergies des neutrons. Le rapport des comptages à ces deux angles donne le rapport des sensibilités pour les énergies correspondantes, à condition de faire une correction pour la variation d'angle solide apparent. En combinant de telles mesures de rapport, pour différents angles et différentes énergies, on arrive à construire toute la courbe, entre $2 \mathrm{MeV}$ et $4,2 \mathrm{MeV}$. La réponse du compteur varie lentement et les résonances du carbone à $2,08 \mathrm{MeV}$, et surtout celle à $2,9 \mathrm{MeV}$ qui est très large, ne produisent pas de perturbation appréciable, contrairement à ce qui se passe avec les « longs compteurs " habituels; ii) entre 0,3 et $2 \mathrm{MeV}$, la variation de l'efficacité a été étudiée en mesurant la distribution angulaire apparente des neutrons de $2 \mathrm{MeV}$ et $2,4 \mathrm{MeV}$ diffusés par l'hydrogène contenu dans un morceau de polyéthylène. La distribution angulaire est isotrope dans le système du centre de masse, il s'ensuit que, dans le système du laboratoire, le flux de neutrons diffusés varie comme le cosinus de l'angle $\varphi$ de déviation des neutrons, et que l'énergie des neutrons diffusés varie comme $\cos ^{2} \varphi$. On peut, en comparant cette loi théorique et la distribution mesurée, obtenir la variation de sensibilité du compteur en fonction de l'énergie. Pour chaque valeur de $\varphi$, on mesurait aussi la diffusion provoquée par un échantillon de graphite, pour évaluer la contribution du carbone contenu dans le polyéthylène. Les corrections de diffusion multiple ont été calculées au moyen d'un code de Monte-Carlo approprié. Le résultat ne dépasse pas une précision globale de $\pm 10 \%$. Aucune variation significative d'efficacité n'est visible dans ces conditions entre 0,3 et $2 \mathrm{MeV}$.

II.3. ÉTUDE DU BRUIT DE FOND. - Il se compose : i) d'un bruit de fond intrinsèque dû partiellement au rayonnement cosmique, son intensité est de $4,5 \mathrm{cps} / \mathrm{mn}$; ii) d'une contribution proportionnelle à la source de neutrons à mesurer. Il s'agit essentiellement de neutrons qui traversent la protection, soit en provenant de la source, soit après une diffusion sur les parois de la salle. On mesure cette contribution en interposant devant l'entrée du collimateur une barre de métal de longueur suffisante pour que sa transmission soit négligeable. Le résultat dépend évidemment de l'énergie : à $300 \mathrm{keV}$, ce bruit de fond est de $0,2 \%$; à $2,7 \mathrm{MeV}$, il est de $4,5 \%$. En augmentant les dimensions de la cuve jusqu'à $1,20 \mathrm{~m}$ de côté, on obtient une amélioration assez substantielle, puisqu'à $2,7 \mathrm{MeV}$ le bruit de fond tombe à $0,3 \%$.

II.4. Résultats des mesures. - L'ensemble de ces mesures permet de définir l'efficacité du système à $\pm 4 \%$ près entre $0,1 \mathrm{MeV}$ et $4,3 \mathrm{MeV}$. Le tableau I donne quelques valeurs de l'efficacité.

III. Etude théorique. - Un programme de MonteCarlo très détaillé a été écrit pour calculer l'efficacité de ce système. La validité des hypothèses utilisées dans le calcul du ralentissement et de la diffusion des neutrons a été testée en comparant la distribution des neutrons thermiques calculée avec une distribution expérimentale. La dépendance en énergie d'efficacité ainsi calculée correspond à peu près avec les résultats expérimentaux, mais la valeur absolue est trop forte de $30 \%$ environ.

\section{BIBLIOGRAPHIE}

[1] FORT (E.) et LERoy (J. L.), Conf. données nucl. microscopiques, A.I.E.A., CN 23/67, 1966.

[2] Theus (R. B.), McGarRy (W. I.) et Beach (L. A.), Nucl. Phys., 1966, 80, 273.

[3] Axton (E. J.), Cross (P.) et Robertson (J. C.), J. Nucl. Energy, A/8, 1965, 19, 409. 